\title{
FACILITY LOCATION IN LOGISTIC NETWORK DESIGN USING SOFT COMPUTING OPTIMIZATION MODELS
}

\author{
Shaju Varughese ${ }^{1}$ and Gladston Raj $\mathrm{S}^{2}$ \\ ${ }^{1}$ Scholar, Bharathiar University, Coimbatore, Tamilnadu, India \\ ${ }^{2}$ Head of Department of CS, Govt. College, Nedumangadu, Thiruvananthapuram, \\ Kerala, India.
}

\begin{abstract}
Discovery of the optimal best possibility of location for facilities is the central problem associated in logistics management. The optimal places for the distribution centres (DCs) can be based on the selected attributes that are crucial to locate the best possible locations to increase the speed of the facility service and thus reduce the overall transport cost and time and to provide best service. The major task is to identifying and locating the required number of DCs and its optimum locations are considered as the important goals for the design of any logistics network. The number of DCs will clearly depends upon many factors like population, capacity of the facility, type of facility etc. but locating the optimum locations of DCs will reduce the overall cost. But, for solving such a wide problem space, the powerful tools are the soft computing based approaches and that are well suited and find a meaningful solution in finite time. In this work, we are going to find the optimum locations of DCs for logistics using various soft computing methods.
\end{abstract}

\section{KEYWORDS}

Logistic, Heuristic, Hybrid, Inbounded, Crossover, Mutation, Simulated, Annealing, Direct Search.

\section{INTRODUCTION}

The facility location analysis is the process identifying the location problem and generates a common model for finding the optimal location[22]. This is nothing but identify $\mathrm{k}$ center problem, which deal with the optimal placement of facilities to minimize transportation costs by satisfying some constraints[22]. The techniques also apply to cluster analysis so that the clustering algorithms used in data mining can also be applied for location analysis[22]. In this work, using the basic concept various soft computing based models are developed for finding the optimum location for facility location for logistics analysis.

\subsection{Facility Location Problem (FLP)}

Facility Location problem in logistic management is the process of locating and allocating optimal location to construct one or more facilities in order to utilize the largest set of users within a specified service distance. The facility location problem is a challenging and non-linear problem in the areas of production, procurement, distribution, operations management and combinatorial optimization. The problem finds an optimal location of facilities considering facility construction costs, transportation costs, etc. This problem is very popular because it is faced by majority of companies. A large number of researchers have studied this problem and proposed different approaches as solution. 
Location models are very difficult to solve, especially for large heterogeneous problem instances[3]. There are a number of specific tools that are available in the operational industry that overcome the tedious computational complexity of a location models. Besides, location models are application dependent. Their objectives, constraints and variables are determined by particular problem under study. So, it is very difficult to develop a common model that can customize to a particular location model that is optimal for all potential or existing applications [22].

There are two types of techniques in general[3]

- Exact Solution Techniques[3]

- Heuristic Solution Techniques[3]

\subsection{The FLP and Logistics}

\subsubsection{Logistic}

Logistic is a framework and it is essentially a planning orientation that seeks to create an efficient planning for the flow of products and required information through a business. The traditional orientation was to perform each functional task as well as possible with limited consideration given to how one work area impacted another[22].

Optimization is the process of minimizing or maximizing the output of a well defined functions model. Facility-location problem is the problem of locating facilities and its analysis deals with existing facilities, if available, and clients in order to optimize several economic criteria. This problem tries to satisfy the fixed, presumably known demands of $m$ customers by supplying each customer with the same commodity from a facility to be established at a particular site $j$ [22]. It is assumed that there are $\mathbf{n}$ candidate sites where facilities can be established if necessary. The unfortunate aspect of this very general class of mathematical programming problems is the fact that many of these problems have a high degree of complexity and general purpose algorithms to solve them dependably are not readily available[22].

\subsubsection{Facility Network Design}

Classical economics neglected the importance of facility location in overall network design for efficient business operation is not well defined in classical economics[22]. The economists originally discussed supplier and customer demand relationships, facility location and transportation cost were either nonexistent or equal among competitors. In business management, the number, size, and geographical relationship of service providers used to perform logistical operations directly affect the customer service capability and cost[22].

\subsubsection{Logistics Network}

A logistics network provides cost effective and efficient way of facilitates delivery of packages. It is a network of DCs which can be utilized by multiple organizations[22]. These organizations can share associated logistics costs. Consolidation of a variety of products and truckload transportation help for mitigation of traffic congestion, saving of fuel, and reduction in labour costs[22].

The type of products that can be handled by these DCs include furniture, consumer electronics, paper products and stationary items, drugs, textile products, perishable and non-perishable food products, non-food grocery products, home improvement and hardware items[22]. Since these 
products include almost everything related to domestic and office needs, implementation of the PLN can reduce daily vehicle trips for purpose of shopping by substantial amount[22]. Moreover, not only retailers, wholesalers and manufacturers can use of PLN but third party logistic companies working with cooperative contract, small and mid-sized freight transportation enterprises can collaborate with PLN and reduce the costs with high quality service to their customers[22].

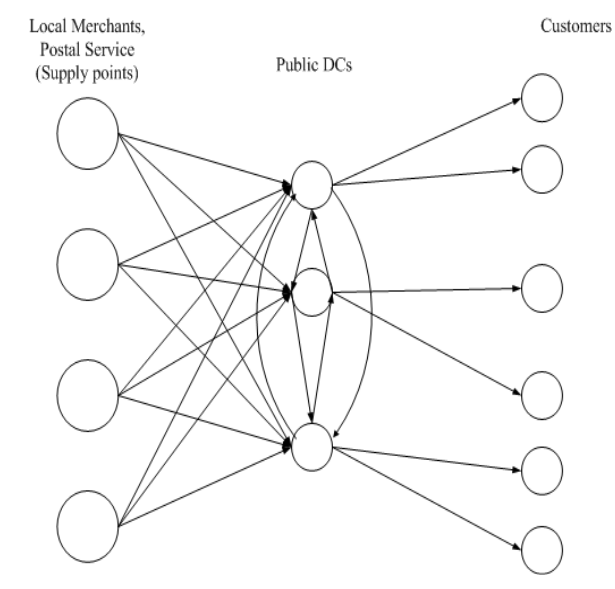

Figure 1. The Package Flow in Logistics Network

\section{BACKGROUND ON FACILITY LOCATION AND ABOUT THIS WORK}

\subsection{Facility Location Problem}

The following are the major criteria for finding the optimal location for facility location problem.

- Capacity of facility.

- Facility construction costs

- Transportation costs, etc.

These problems are the common issues faced by most of the companies. Also, number of research proposals developed and implemented in this matter.

A decision maker or analysis system should build in this case that leads the decision makers to decide the optimal location for a facility.

\subsection{The Proposed Logistics Network Design}

The Public Logistics Network (PLN) for the continental U.S. was based on the underlying road networks and the state level population[2]. This research made use of the basic design approach that was developed by Bansal. The Bansal proposal consists of the generation of the Underlying Road Network (URN), developing the network of public DCs, estimation of average package delivery time, and finding public DC locations that minimize average package delivery time[22].

In this work, we modified and generate a new version of that design that make use of distance as the major metric, instead of using the "average package delivery time" as a metric for optimization, we used simple distance as the metric in the fitness function of the soft computing 
model[22]. This is minimizes the optimization time. Since the distance is directly proportional to package delivery time, we believe that this approach also will lead to equal results, logically with in lesser time[22].

- Structure the Road Network layout

- Design and develop the network for generating the DCs,

- Finding the DC locations that minimize the distance between the DCs and the User locations.

\subsubsection{Road Network of USA}

This map indicates the road network that using US census data set.

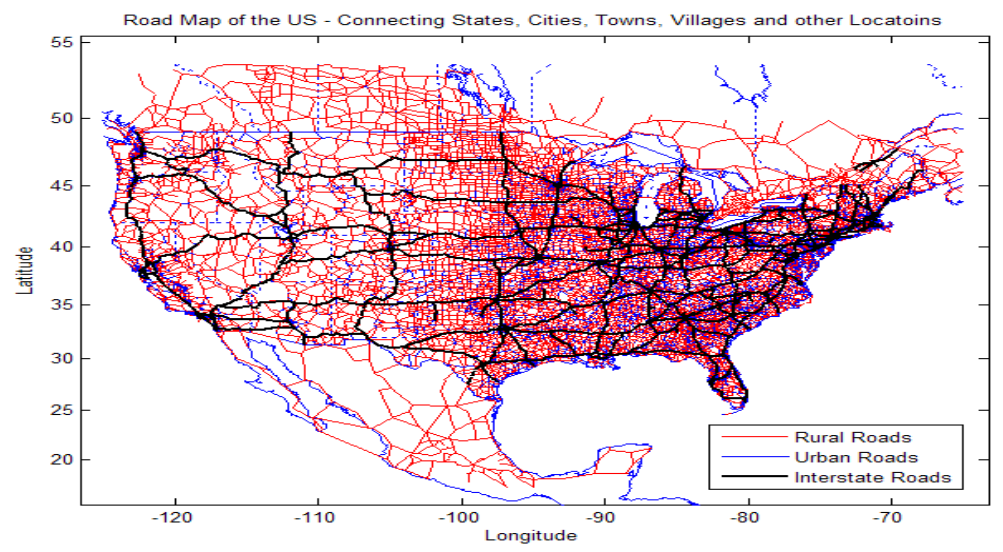

Figure 2. Road Network layout of USA

\subsubsection{Structure the Road Network of Regional Distribution Centers.}

The population in RDC is represented by total 925 U.S. census blocks that are plotted on the map of RDC. A sub-graph of the road network was generated that is then followed by the removal of two-degree nodes from the network. Each point in this network is a potential location for a $\mathrm{DC}[22]$.

The following graph/map shows the road network of Louisiana, USA that will be the example of a sub graph we created and used to create the regional distribution centers (RDC).

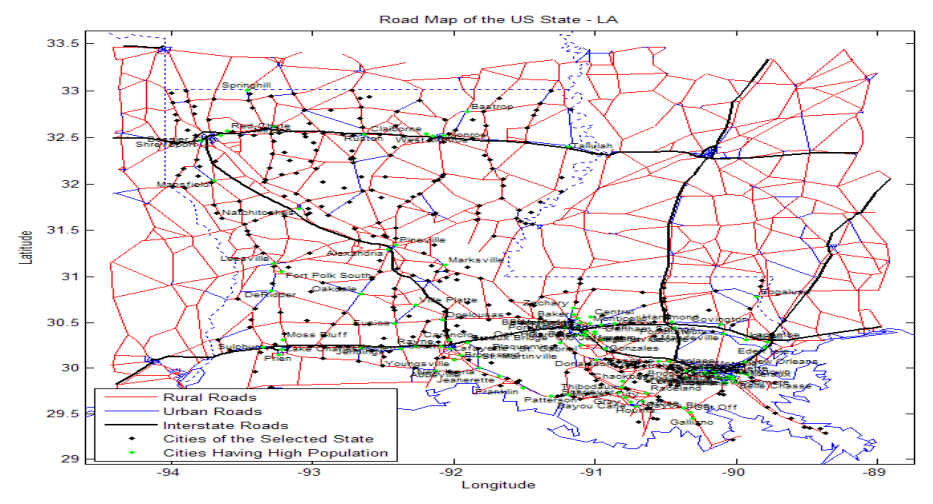

Figure 3. Road Network of RDCs 


\subsubsection{Network of DCs}

If needed, we may also create the network of DCs as follows.

The arcs connecting census blocks to the URN are added to the network and the shortest time paths and distances between each pair of points are calculated using Dijkstra's algorithm[22]. DCs will be located at some of the key points and then connected to each other using Delaunay Triangulation [19] to form a network of public DCs. The shortest time paths between all pairs of DCs is found and those paths and distances are then used to calculate the percent flow of the packages, wij from DC $\mathrm{i}$ to DC $\mathrm{j}$ using order based proximity factors developed by Kay and Parlikad [1] using following equations.

\section{The Soft COMPUTING BASEd FACILITY LOCATION System MODELS}

\subsection{Genetic Algorithm}

Genetic algorithm is a population-based search method. Genetic algorithms are acknowledged as good solvers for tough problems. It is an iterative procedure maintaining a population of structures that are candidate solutions to specific domain challenges. During each temporal increment (called a generation), the structures in the current population are rated for their effectiveness as domain solutions, and on the basis of these evaluations, a new population of candidate solutions is formed using specific genetic operators such as reproduction, crossover, and mutation

The following pseudo code outlines the design of GA for solving Facility Location.

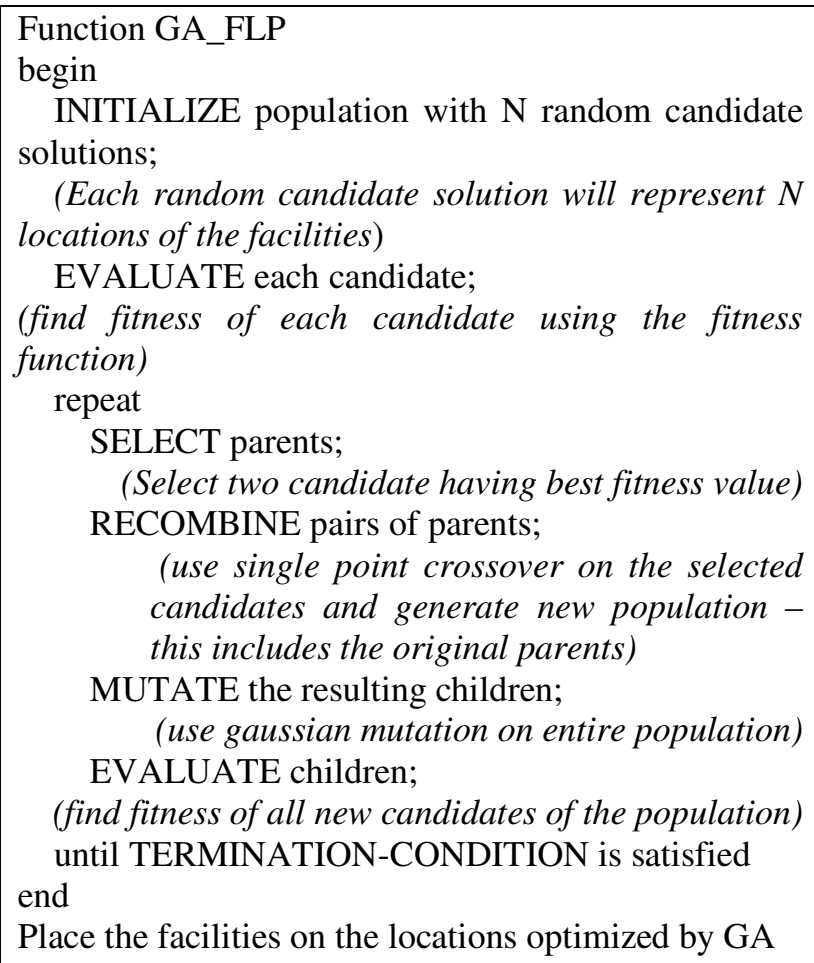

Figure 4. The GA Facility Location Model 


\subsection{The Fitness Function}

The following function is used to find the fitness at the set of facility location $X Y_{F}=\left(X_{i}, Y_{j}\right)$, where $i, j=1$ to $n$. The set of points which has the lowest fitness vaue will be the optimum location for placing facilities. $\mathrm{XY}_{\mathrm{U}}$ is the locations of all the customers(cities, towns, villages)

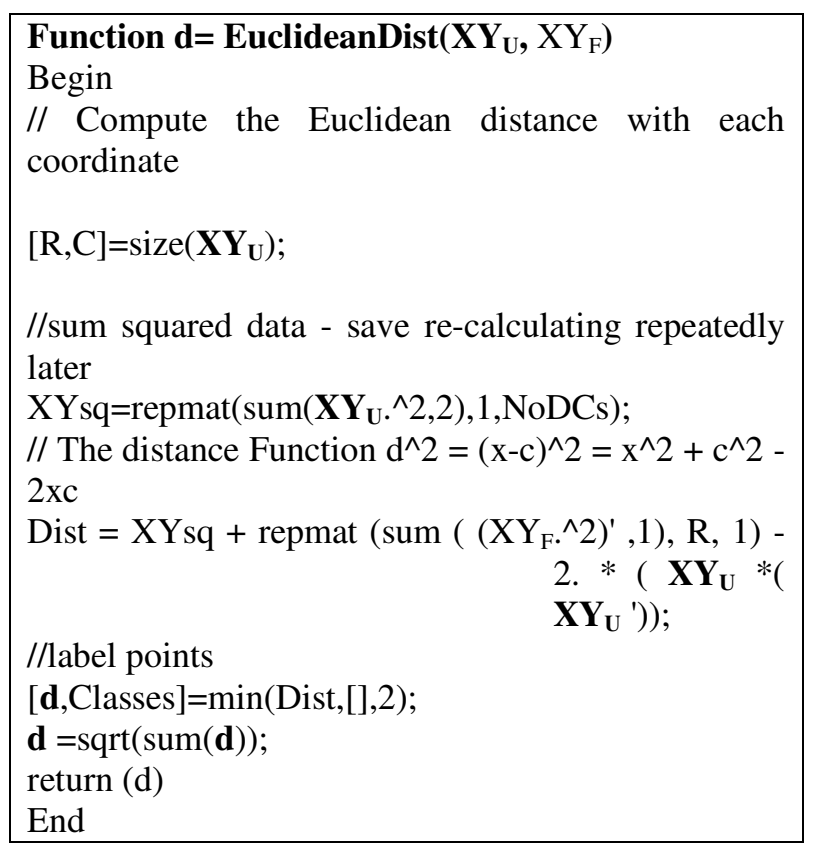

Figure 5. The Fitness Function

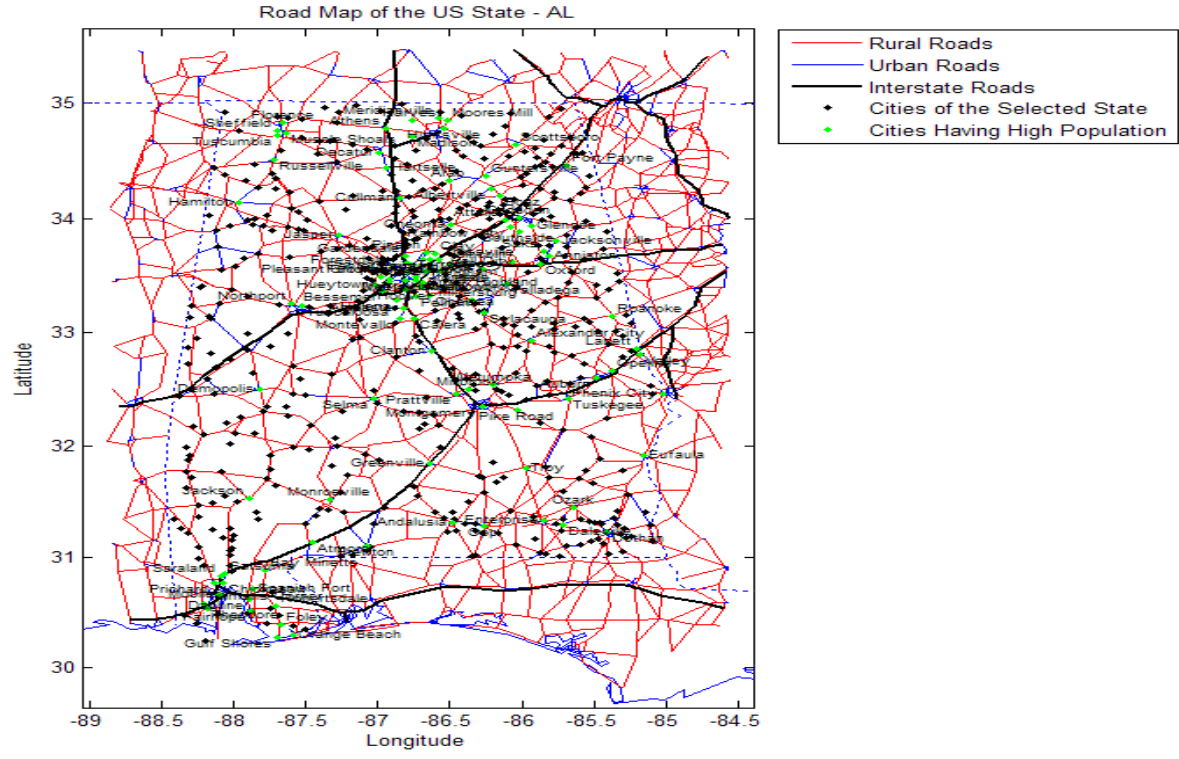

Figure 6 Map : Alabama, USA 
International Journal of Computer Science \& Information Technology (IJCSIT) Vol 9, No 5, October 2017

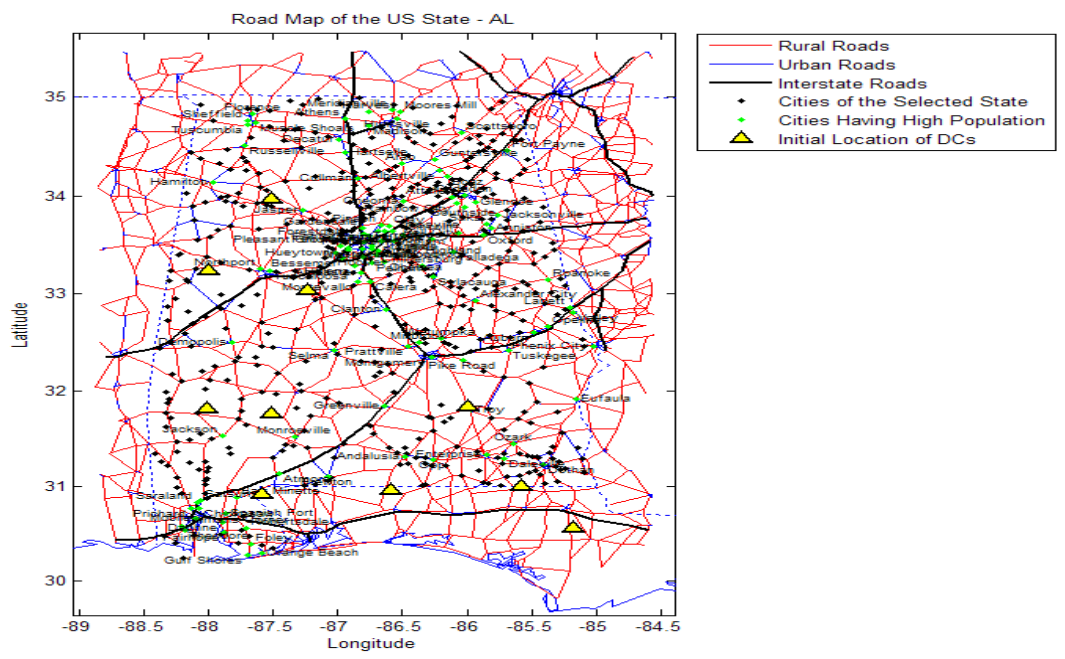

Figure 7: Random Initial Locations of DCs

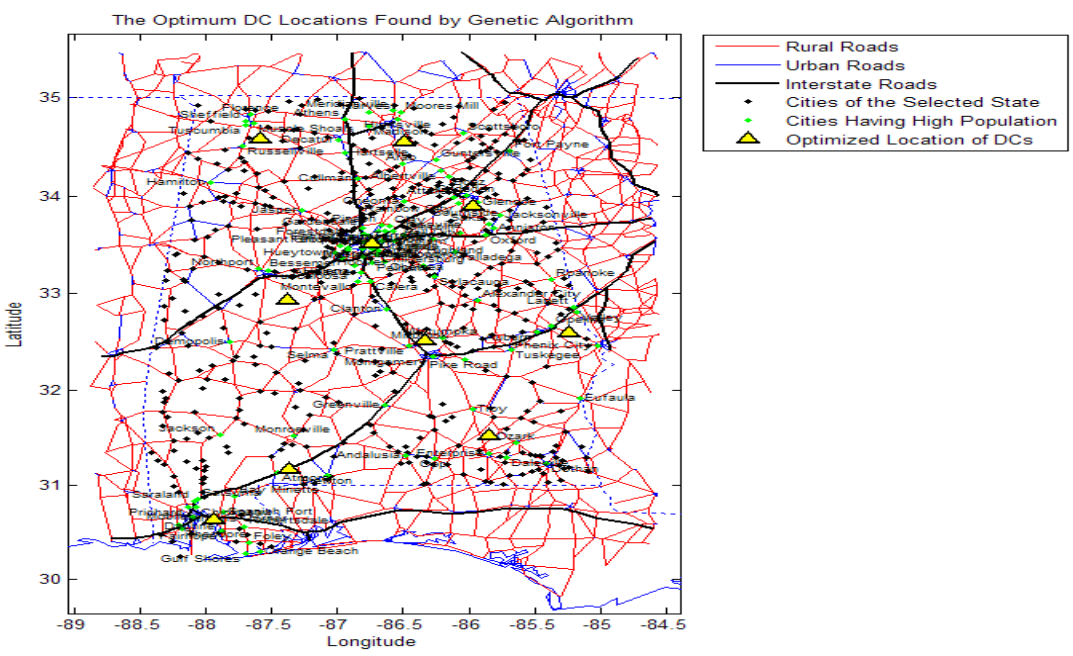

Fiqure 8: Facility Location by Genetic Algorithm

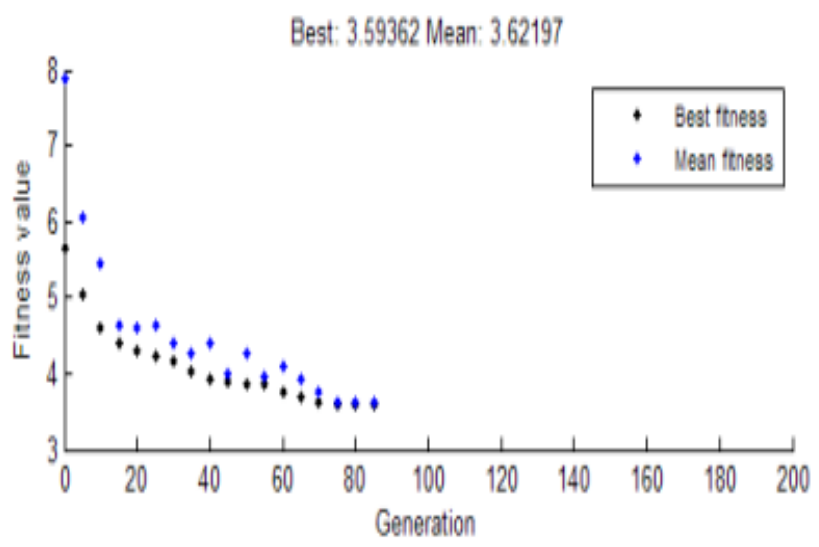




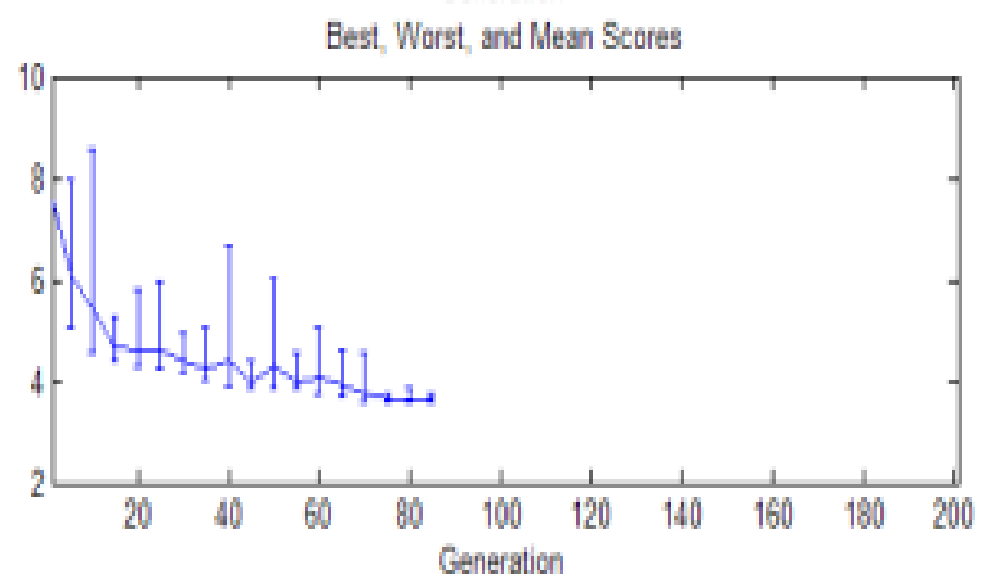

Figure 9: Performance of Genetic Algorithm

\subsection{Simulated Annealing}

The structural change in the materials at the time of cooling is the concept and the working terminology of Simulated Annealing(SA)[22]. The Metropolis algorithm developed in 1953 was the basic structure of Simulated Annealing and it was published. The algorithm in this paper simulated the cooling process of materials and their structural changes from their heavy hot stage by using the heat bath[22]. This cool down process is known as annealing. The working of the algorithm is that in step by step it will allow the material to cool and transform in to a steady solid state.

The basic idea of metropolis algorithm has initiated by Kirkpatrick in 1982. It has been applied to various optimization problems. The basic concept is to find the feasible solution to a problem by using simulated annealing as a major tool and converge to an optimal solution[22].

The law of thermodynamics state that at temperature, st, the probability of an increase in energy of magnitude, $\delta \mathrm{EM}$, is given by $\mathrm{P}(\delta \mathrm{EM})=\exp (-\delta \mathrm{EM} / k s t)$

Where $k$ is a Boltzmann's constant.

Based on the calculated new energy The system has moved to the same state if the new energy has decreased otherwise the new state is accepted using the probability returned by the above formula[22]. A certain number of iterations are carried out at each temperature and then the temperature is decreased. This process is an iteration process and recursive until the system freezes in to a pre-assigned steady state[22]. This equation can make use for generating simulated annealing concept directly. It is a common practice to drop the Boltzmann constant into the equation to experiment with different materials. The acceptable probability for the worse case is defined by the equation

Where

$$
\mathrm{PR}=\exp (-c f / \mathrm{c} t)>\mathrm{rv}
$$

$c f \quad=\quad$ the change in the evaluation function

$c t=$ the current temperature

$r v=$ a value between 0 and 1

Function SIMULATED-ANNEALING(Problem, Schedule) returns a solution state Inputs : $\quad$ Problem, a problem 
International Journal of Computer Science \& Information Technology (IJCSIT) Vol 9, No 5, October 2017

Schedule, a mapping from time to temperature

Local Variables

Next, a node

: Current, a node

$\mathrm{TH}$, a "temperature" controlling the probability of downward steps

Current $=$ MAKE-NODE(INITIAL-STATE $[$ Problem $])$

For temp $=1$ to $\infty$ do

$\mathrm{TH}=$ Schedule[temp $]$

If $\mathrm{TH}=0$ then return Current

Next $=$ a randomly selected successor of Current

$\Lambda \mathrm{E}=\operatorname{VALUE}[$ Next $]-$ VALUE $[$ Current $]$

if $\Lambda \mathrm{E}>0$ then Current $=$ Next

else Current $=$ Next only with probability $\exp (-\Lambda \mathrm{E} / \mathrm{TH})$

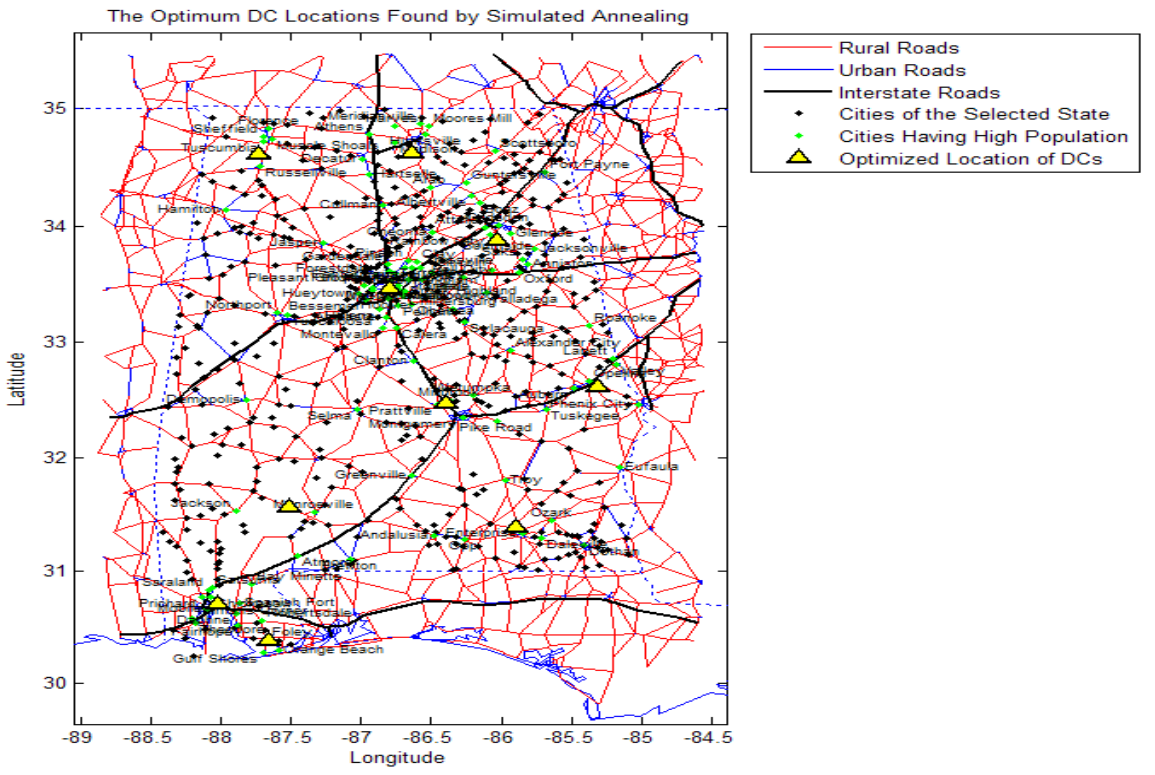

Figure 10 Facility Location by Simulated Annealing
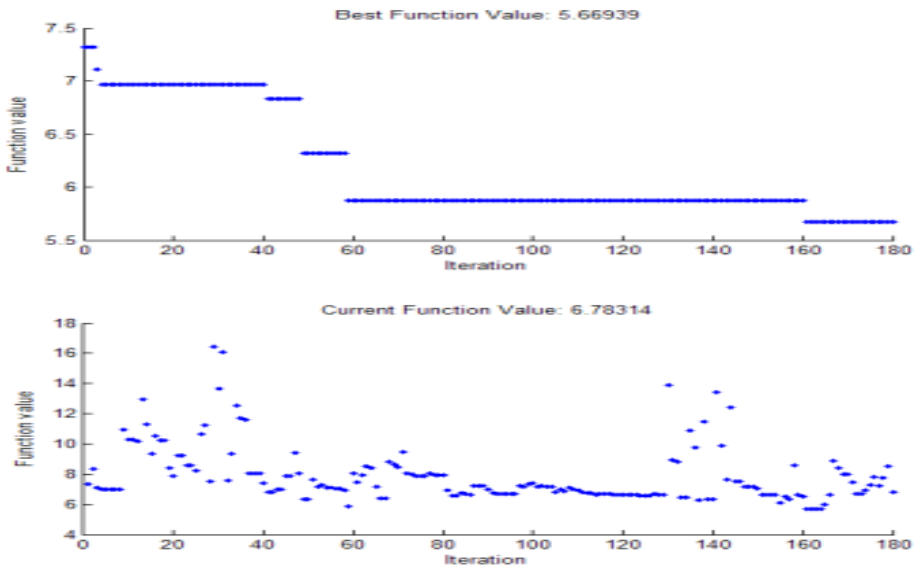

Figure 11 Simulated Annealing performance graph 


\subsection{Direct Search}

The optimal solutions finding procedure in multidimensional spaces is some extend similar to the methods used in the single dimensional space for finding the optimal solution. In multidimensional optimization the computational complexity of the applications depend the type of application, solution method applied and the data extend.

The systematic search and find all possible evaluation procedures and corresponding solutions in the multidimensional space and select the maximum or the minimum optimization solution based on the objective. This is a very generally applicable approach and may even be useful if the solution space is relatively small. However, as the dimensions of the problem space, (number of independent variables), increase, the computational complexity of this solution approach quickly becomes unmanageable. Therefore, apply the methods for finding the optimal solution by applying the efficient form of search in the solution space and these are need not be in entire solution space.

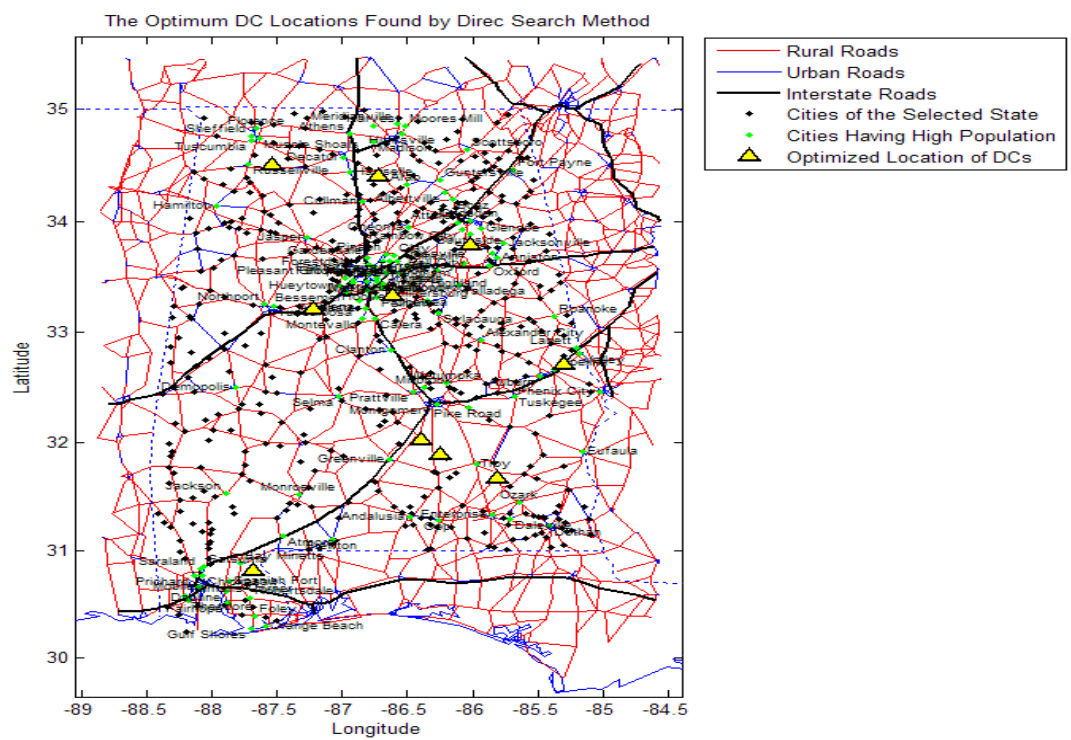

Figure 12: Facility Location based on Direct Search method 
International Journal of Computer Science \& Information Technology (IJCSIT) Vol 9, No 5, October 2017
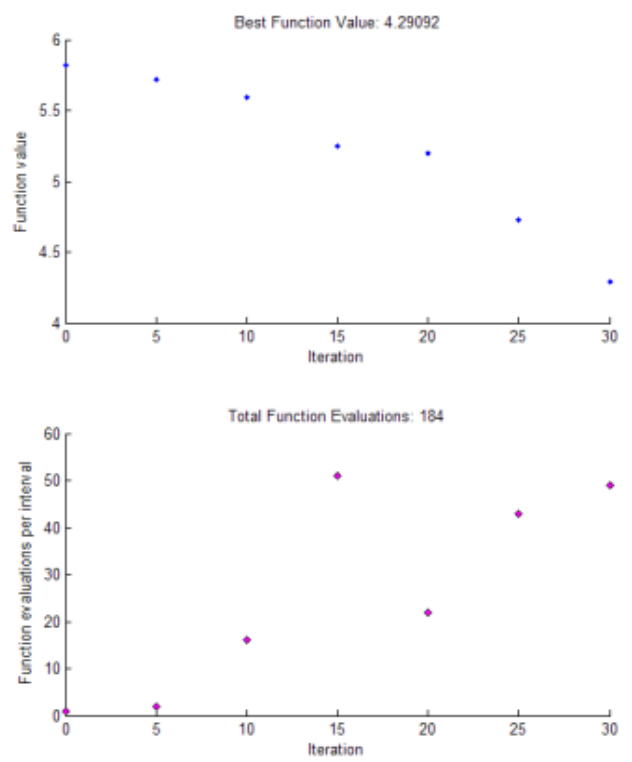

Figure 13: Performance layout of Direct Search

\subsection{K-means Clustering}

Simply put, k-Means Clustering is an algorithm among several that attempt to find groups in the data. In pseudo code, it follow this procedure:

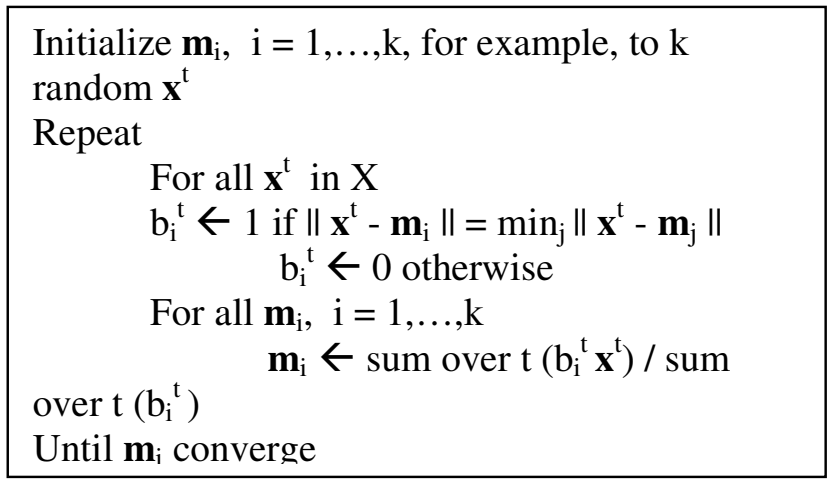

The vector $\mathbf{m}$ contains a reference to the sample mean of each cluster. $\mathbf{x}$ refers to each of our examples, and $\mathbf{b}$ contains our estimated class labels.

Explained perhaps more simply in words, the algorithm roughly follows this approach: Choose some manner in which to initialize the $\mathrm{m}_{\mathrm{i}}$ to be the mean of each group (or cluster), and do it.

For each example in your set, assign it to the closest group (represented by $\mathrm{m}_{\mathrm{i}}$ ). For each $\mathrm{m}_{\mathrm{i}}$, recalculate it based on the examples that are currently assigned to it. Repeat steps 2-3 until $\mathrm{m}_{\mathrm{i}}$ converge. 


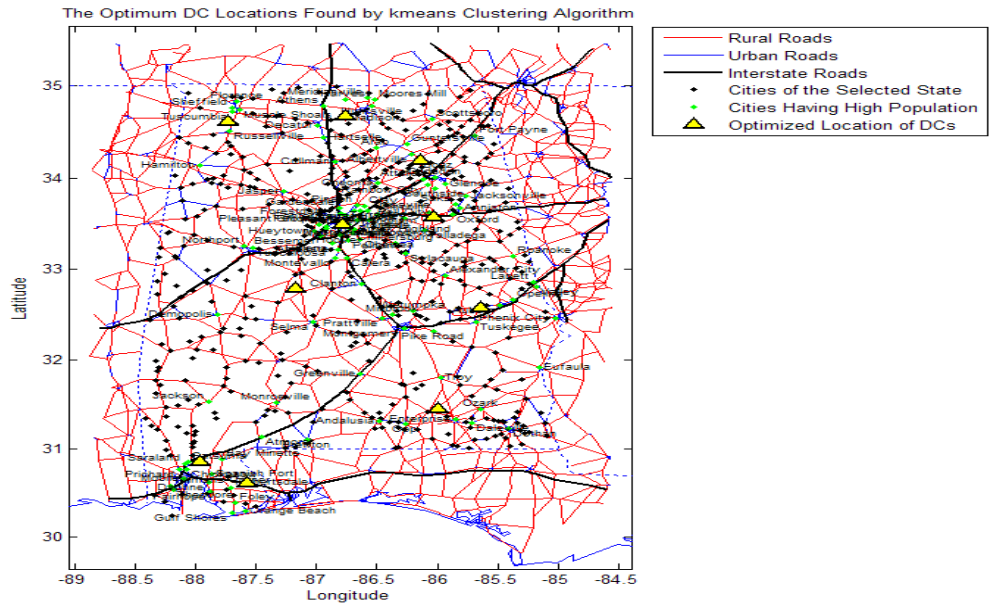

Figure 14: K-means clustering Algorithm based facilities

\section{RESUlT AND DISCUSSION}

We have implemented the proposed soft computing based models for facility location in logistics analysis using Matlab software version R2012s. We used some of the functions of Logistics Engineering Toolbox "Matlog Version 16" in this research. We tried to use almost equal input parameters for each and every evaluated method. We used the USA census data and map data which is much suitable for this kind of research. We decided to use USA data because, it is the only data refereed in some of the previous works and there seems no such detailed data available for any other country for validating the methods of facility location and logistics analysis.

\section{The basic data set parameters of the Soft Computing Models}

Genetic Algorithm options

StallGenLimit : $\quad 20$,

Generations : $\quad 200$

PopulationSize : $\quad 20$

CrossoverFen : $\quad$ Two Point Crossover

MutationFen : Gaussian

Simulated Annealing Options

MaxIter : 200

MaxFunEvals $\quad: \quad 200$

InitialTemperature $\quad: \quad 100$

AnnealingFen : $\quad$ Fast Annealing

HybridFen : fminsearch

TemperatureFcn : temperatureexp

Pattern Search Options

MaxIter : 200

MaxFunEvals : $\quad 200$

InitialMeshSize : $\quad 1.0 \quad$ MADSPositiveBasis

SearchMethod' : $\quad$ ADSPositiveBasis2N

k-means Clusterign

MaxRepetitions : $\quad 200$

MaxIter : 20 
The following table shows the overall results of this work. Since the performance of a soft computing model will depend up on several factors, and some random conditions, we run each algorithms several times and only selected the values which are minimum.

Table 1 - The Overall Performance

\begin{tabular}{lll}
\hline $\begin{array}{l}\text { Facility } \\
\text { Method }\end{array}$ & $\begin{array}{l}\text { Location Avg. } \\
\text { Distance }\end{array}$ & $\begin{array}{l}\text { Time } \\
\text { Consumed }\end{array}$ \\
\hline $\begin{array}{ll}\text { Random } \\
\text { Location }\end{array}$ & 9.44 & - \\
GA & 3.59 & 2.63 \\
SA & 5.67 & 5.13 \\
DS & 4.29 & 1.44 \\
k-means & 3.95 & 3 \\
\hline
\end{tabular}

The following graph shows the performance of the four algorithms in terms of the minimum average distance achieved. The average distance is the average of distance between all DCs and the Customer locations. Each Customer is bound with a nearest DC and the distance between each customer to that corresponding DC is calculated and then the average of all such distances were calculated. In this graph, the first column shows the reference distance which is nothing but the initial average distance of DCs that are randomly placed on the map.

With respect to the average distance, the GA based model performed well. It means, GA found the optimum facility locations better than the other compared methods.

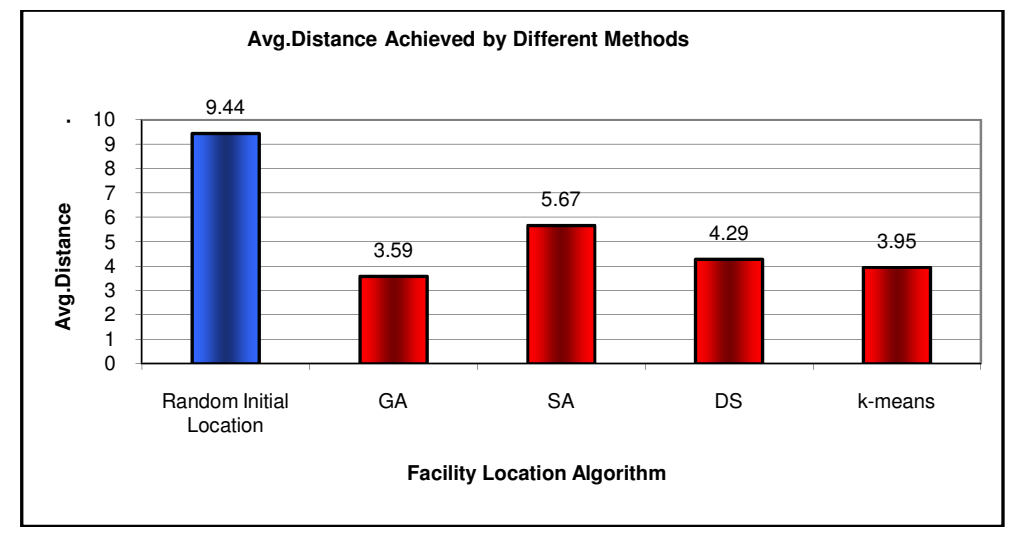

Figure 15. The Performance in Terms of distance

The following graph shows the performance in terms of cpu time. Even though direct search consumed lesser time, it doesn't fine much optimum locations like GA. We have to note that the time in the case of GA,SA and DS includes some additional time that is spent of displaying/updating some of the performance graphs also (without those performance graphs, there will be much reduction in time in GA, SA and DS) 


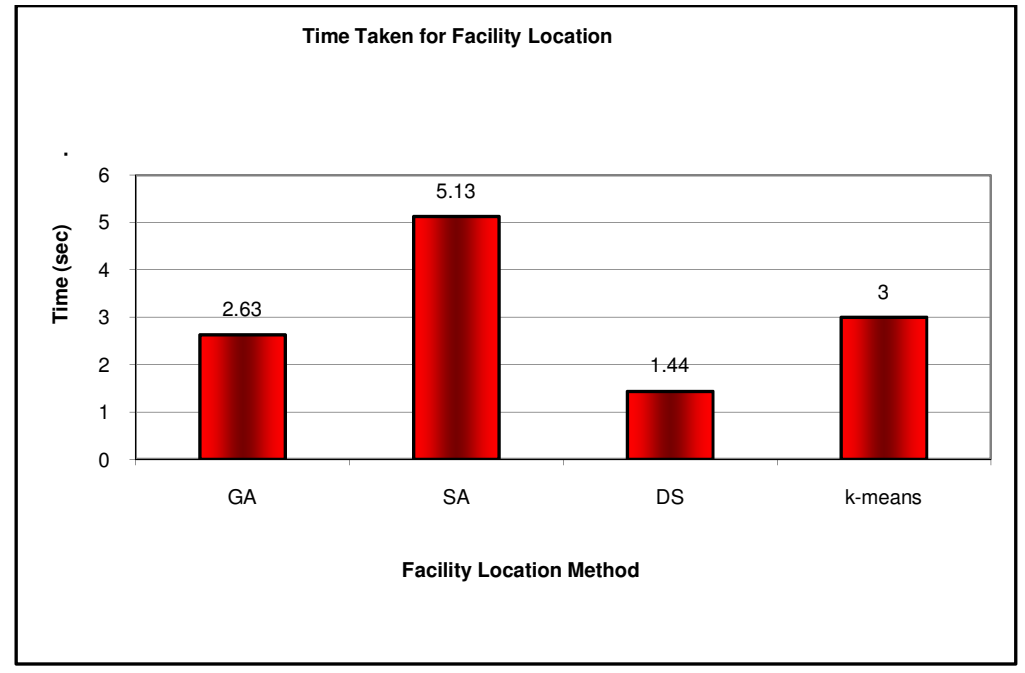

Figure 16. The Performance in Terms of CPU Time

\section{CONCLUSION}

The major issue faced logistic management is none other the facility location. Also, it is a one of the leading research area. In this work we addressed the possibilities and its performance using soft computing based models for facility location in for logistics. We used soft computing based clustering approach is the basic facility Location Problem \& Logistic Analysis.

As per the performance and dataset based results, soft computing based optimization models successfully found optimum locations of facilities in considerably meaningful time limit.In this work, we used a simple Euclidean distance function as the major fitness function in the design of soft computing based location optimization model. But, there are much more constraints and parameters in a practical logistics problem that can be included in the design of the fitness function such as (1) travel time with respect to road type, (2) Collection and distribution time at DCs, (3) Capacity of the facility (4) Facility type ( seasonal or not). This kind of more constraints and parameters can be included in future design of soft computing based optimization models. Our future works will address these issues.

We have designed the proposed models as a single objective problem. But there may be the situation where facility location and logistics analysis should optimized based on more than one objective during optimization. Future works may address the design of soft computing based optimization models for multi objective optimization scenarios.

\section{REFERENCES}

[1] Erlenkotter, D., "A Dual-Based Procedure for the Uncapacitated Facility Location." Operations Research, Vol 26(6), pg. 992-1009, 1978.

[2] Francis, R.L., McGinnis, F.L., Jr., White, J.A., "Facility Layout and Location: An Analytical Approach." Prentice Hall, 2nd Ed., 1974.

[3] Juliana Karakaneva, "A LOCATION PROBLEM MODELING AND SOLVING", Trakia Journal of Sciences, Vol 1, No 4, pp 1-7, 2003,ISSN 1312-1723, Copyright (C) 2003 Trakia University

[4] HK Smith, G Laporte and PR Harper, "Locational analysis: highlights of growth to maturity", http://eprints.soton.ac.uk/68930/1/Locational_Analysis_-_Smith,_Laporte_and_Harper.doc

[5] Michael J. Bucci, Michael G. Kay, Donald P. Warsing†, Jeffrey A. Joines, "Metaheuristics for Facility Location Problems with Economies of Scale", IIE Transactions 
[6] Michael J. Bucci, Ryan Woolard, Jeffrey Joines, Kristin Thoney, Russell E. King, "An Application of Heuristics Incorporating Economies of Scale to Facility Location Problems in Carpet Recycling"

[7] Biehl, M., Prater, E., Realff, M.J., 2007, Assessing performance and uncertainty in developing carpet reverse logistics systems, Computers and Operations Research, 34, 443-463.

[8]. Brimberg, J.,Hansen, P., Mladenovic, N.,Taillard, E.D. ,2000, Improvements and Comparison of Heuristics for Solving the Uncapacitated Multisource Weber Problem, Operations Research, 48, $444-$ 460.

[9] Bucci, M.J., Kay, M.G., Warsing, D.P., Joines, J.A., 2009, Metaheuristics for Facility Location with Economies of Scale, Fitts Department of Industrial and Systems Engineering working paper, North Carolina State University, Raleigh NC.

[10] Cooper, L., 1963, Location-allocation problems, Operations Research, 11, 331-343.

[11]CARE (Carpet America Recovery Effort) annual report, 2007, http://www.carpetrecovery.org/pdf/annual_report/07_CARE-annual-rpt.pdf

[12]CARE(Carpet America Recovery Effort) network website, 2009, http://www.carpetrecovery.org/pdf/reclamation_centers/Carpet_Reclamation_Center s.pdf

[13] Daskin, M.S.,1995, Network and discrete location: models, algorithms, and applications, John Wiley and Sons, New York.

[14] de Brito, M.P., Dekker, R., Flapper, S.D.P., 2003, Reverse Logistics - a review of case studies, ERIM Report Series.

[15] Fleishchmann, M., Krikke, H.R., Dekker, R., Flapper, S.D.P., 2000, A characterization of logistics networks for product recovery, Omega, 28, 653-666.

[16] Louwers, D., Kip, B.J., Peters, E., Souren, F., Flapper, S.D.P., 1999, A facility location allocation model for reusing carpet, Computers and Industrial Engineering,

[17] Mirchandani, P.B., Francis, R.L., 1990, Discrete Location Theory, Wiley, NewYork.

[18] Realff, M.J., Ammons, J.C., Newton, D., 1999, Carpet Recycling: Determining the 38:3, 547-567.

[19] Realff, M., Systems Planning for Carpet Recycling. 2006, Recycling in Textiles, Editor Youjiang Wang, CRC Press.

[20] Repa, E.W., 2005, NSWMA's 2005 Tip Fee Survey, National Solid Wastes Management Association Website,://wastec.isproductions.net/webmodules/webarticles/articlefiles/478-

Tipping\%20Fee\%20Bulletin\%202005.pdf

[21] Shaju Varghese, Gladston Raj S, "A Genetic Algorithm Based Optimization Model for Facility Location in Logistic Network Design”, International Journal of Applied Engineering Research, Vol 10, No. 69, pp-338-344, 2015, ISSN 0973-4562.

[22] Shaju Varghese, Gladston Raj S, "Simulated Annealing and Direct Search Based Optimization Models for Facility Location in Logistic Network Design" International Journal of Computer Applications, Vol 132, pp- 31-37, December 2015, ISSN: 0975-8887

\section{Authors Profile}

Mr. Shaju Varghese received his M.Sc. (Maths), M.C.A., and M.Phil. in computer Science. Now working as Head of the Department of Computer Applications at Baselios Poulose II Catholicos (B. P. C ) College, Piravom, Kerala, India. He was the Principal Investigator of the Minor Research Project "Computerized Facility Location Analysis In Rural Area Using Clustering", 2010, funded by Universities Grant Commission, India. His research interest includes Data Mining, Facility Location Problem, and Cyber Criminology, and has four international Journal publications in related to his research area. Currently he is pursuing Ph.D. in Computer Science at Bharathiar University, Tamilnadu, India.

Dr. Gladston Raj S. received his M.Sc (CS), M.Tech (Image Computing) and PhD in Computer Science from University of Kerala and Completed UGC-NET from University of Kerala and PGDCH (Computer hardware) from MicroCode, $\mathrm{He}$ is Now working as Head of the Department of Computer Science at Govt. College Nedumangad, Kerala, India. His area of interest includes Image Processing, Signal Processing, Datamining. He is providing research guidance for Ph.D scholars from different areas of research and has presented several invited talks in this areas
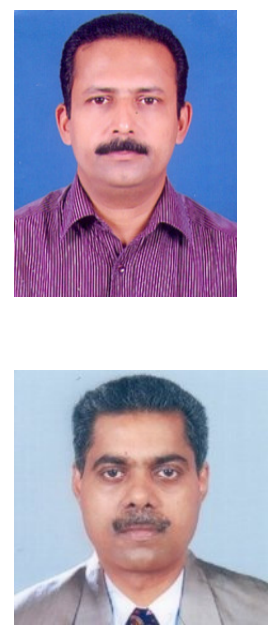